\title{
Biosparging: efficacia ed economicità per i siti contaminati da idrocarburi
}

\author{
Rosanna Feola ${ }^{1}$ \\ ${ }^{1}$ Tecnologie per l'ambiente
}

\begin{abstract}
La cattiva gestione delle attività antropiche è una fra le principali cause della contaminazione delle matrici ambientali. Negli ultimi anni, Il crescente interesse per l'ambiente e per la sostenibilità ha portato alla necessità di adottare nuovi approcci e favorire la bonifica delle matrici ambientali. Tra i processi disponibili, trova applicazione la bioremediation ed in particolare il biosparging che, tramite processi di natura biologica, permette la degradazione dei composti indesiderati nell'ambiente. Il biosparging è particolarmente adatto per la rimozione degli idrocarburi. II presente studio riporta l'analisi di un caso studio di un sito contaminato da idrocarburi policiclici aromatici (IPA) e idrocarburi di petrolio (TPHs), in cui, grazie all'utilizzo combinato di aria e specifici bioattivatori e nutrienti, si sono ottenute alte efficienze di abbattimento sia nel terreno che in falda.
\end{abstract}

\section{Inquadramento normativo e proposta d'intervento}

La presenza di attività commerciali ed industriali, lo smaltimento incontrollato dei rifiuti, I'utilizzo sempre maggiore di fertilizzanti e pesticidi in agricoltura sono la causa dell'alterazione delle caratteristiche qualitative delle matrici ambientali, (Fig.1). Tale alterazione nel suolo, sottosuolo e nelle acque sotterranee può rappresentare un rischio per la salute umana; diventa dunque fondamentale agire prontamente ed attuare trattamenti di bonifica ${ }^{1}$. L'European Commission Joint Research Centre ha eseguito diversi studi per quantificare il numero di siti europei contaminati o potenzialmente contaminati e le principali fonti di contaminazione ${ }^{2}$. In Italia, I'attuale riferimento normativo è il D.Igs. 152/2006 che alle parte IV, titolo $\mathrm{V}$ stabilisce procedure, criteri e modalità di bonifica, e inoltre distingue i siti potenzialmente contaminati dai siti contaminati. Quando la concentrazione di almeno uno dei contaminanti supera la concentrazione soglia di contaminazione Csc, il sito è definito potenzialmente contaminato 
ed è quindi necessaria una caratterizzazione e un'analisi di rischio sito specifica; nel caso di superamento anche delle concentrazioni soglia di rischio Csr, il sito è detto contaminato ed è pertanto necessaria la messa in sicurezza e/o la bonifica. Nel caso studio che si presenterà, in particolare, si è riscontrata la contaminazione da idrocarburi leggeri e pesanti nel terreno, e da BTEX (Benzene, Toluene, Etilbenzene e Xilene) e IPA (idrocarburi policiclici aromatici) nelle acque sotterranee limitrofe al sito ${ }^{3}$. La contaminazione del sito è stata accertata dall'ARPA locale, ed è stata causata della rottura di una tubazione di adduzione di gasolio presso un'area di rifornimento carburanti. Date le dimensioni contenute dell'area, inferiori a $1000 \mathrm{~m}^{2}$, si è adottata una procedura semplificata ai sensi dell'art. 249 D.Lgs 152/06 ed individuati i limiti tabellari Csc, come obiettivi di bonifica. Sebbene le possibilità di intervento siano molteplici ${ }^{4},{ }^{5}$, al fine di privilegiare tecniche adeguate alle caratteristiche del sito, che riducano in modo permanente la contaminazione, che si possano applicare in situ in modo da ridurre la movimentazione, e che minimizzino i costi di gestione a lungo termine è stato adottato l'intervento del biosparging.

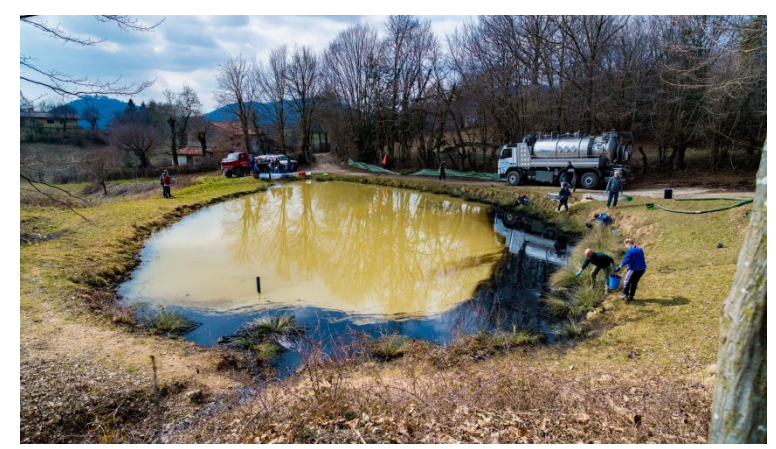

Figure 1: Contaminazione matrice ambientale

\section{Biosparging: tecnologia e applicazione}

Il biosparging è una tecnologia di bioremediation in situ che utilizza microorganismi autoctoni per biodegradare i componenti organici nella zona satura ${ }^{6}$. Essa consiste nell'iniezione di aria (o ossigeno) e sostanze nutritive, se necessarie, per aumentare l'attività biologica dei microrganismi presenti e favorire la biodegradazione di alcuni contaminanti. Il biosparging è particolarmente adatto a ridurre concentrazioni di componenti del petrolio disciolti nelle acque sotterranee, nelle frange capillari e adsorbite al terreno al di sotto della falda acquifera ${ }^{7},{ }^{8},{ }^{9}$. Inoltre, in particolari condizioni, esso è efficace anche per la rimozione di sostanze probabilmente cancerogene come NDMA ${ }^{10}$ e Diossano $1,4^{11}$. Per la zona parzialmente 
satura, si adotta un processo analogo che prende il nome di bioventing ${ }^{12}$, $13,{ }^{14}$. Il processo di biosparging è simile all'airsparging: entrambi prevedono l'iniezione d'aria nel terreno, tuttavia, l'airsparging rimuove i componenti favorendone la volatilizzazione, il biosparging ne promuove la biodegradazione. In presenza di componenti volatili, il biosparging è spesso combinato con il Soil Vapor Extraction, SVE, che, attraverso una serie di pozzi di estrazione, genera una pressione negativa nella zona parzialmente satura e li elimina.

Il biosparging è spesso utilizzato in siti contaminati da prodotti del petrolio con peso molecolare medio (ad es. gasolio, carburante per jet); invece per i prodotti petroliferi più leggeri (ad es. benzina), che tendono ad essere rimossi più velocemente per volatilizzazione, si applica l'airsparging. Per i prodotti più pesanti (ad es. Oli lubrificanti) i tempi di biodegradazione sono maggiori, quindi bisogna fare adeguate considerazioni in funzione degli obiettivi e dei costi di bonifica.

II caso studio presentato riscontrata la contaminazione di $364 \mathrm{~m}^{2}$ della matrice suolo e sottosuolo e di $634 \mathrm{~m}^{2}$ della falda a causa della rottura di una tubazione di adduzione di gasolio. Le concentrazioni sono risultate pari a $496 \mathrm{mg} / \mathrm{kg}$ di idrocarburi leggeri, $12024 \mathrm{mg} / \mathrm{kg}$ di idrocarburi pesanti e $671000 \mu \mathrm{g} / \mathrm{l}$ di idrocarburi totali. Definiti gli obiettivi della bonifica, si è preferito il biosparging all'airsparging, in quanto risulta essere particolarmente vantaggioso poché utilizza attrezzature di semplice installazione, prevede bassi tempi di trattamento (da 6 a 24 mesi), costi competitivi, è eco-friendly ed essendo una tecnologia in situ, crea minimo disturbo. Inoltre, a differenza dell'airsparging, ha un campo maggiore di applicabilità e utilizza un minore flusso d'aria, con conseguenti minori costi di trattamento dei vapori esausti.

Affinché il processo di biosparging sia efficace, bisogna effettuare diverse considerazioni. In una fase di screening iniziale è necessario valutare la permeabilità del suolo e la biodegradabilità dei contaminanti. I costituenti del petrolio sono generalmente biodegradabili in presenza di un adeguato rifornimento di ossigeno, invece maggiore attenzione deve essere posta per I'analisi della stratificazione del suolo. La presenza di materiali a grana fine e l'eterogeneità dei terreni causano un decremento dell'efficienza del processo poiché ostacolano il flusso di aria. D'altro canto, la permeabilità può anche essere ridotta a causa della presenza di ferro, che precipita quando ossidato dall'ossigeno immesso. È poi fondamentale valutare il tasso di crescita batterica che è funzione della temperatura e del pH e cambia a seconda del tipo di microorganismi presenti nel suolo. A tale proposito, risulta fondamentale prelevare campioni e fare analisi di laboratorio, tenendo conto che la popolazione minima necessaria affinché ci sia biodegradazione, è di $10^{3} \mathrm{UFC} / \mathrm{g}$. Nel caso non fosse disponibile, potrebbero essere introdotte colture microbiche ed anche nutrienti per favorire il processo e la crescita cellulare. Nel caso in esame, per migliorare l'efficienza di trattamento sono 
stati aggiunti microorganismi bioattivatori naturali e specifici (conformi alla Classe 1 E.F.B. e all'Allegato 3 alla parte IV del D.lgs 152/2006) biofissati a supporti minerali (sali di dolomia e mordenite) per aumentare i tempi di contatto ${ }^{15}$.

Al fine di dimensionare il processo di biosparging è necessario fare studi pilota. In questo modo, in funzione del raggio di influenza (massima distanza alla quale il flusso d'aria arriva e favorisce la biodegradazione) si determina il numero di punti in cui iniettare l'aria, la sua portata e la sua pressione. È poi necessario definire l'orientamento e il posizionamento dei pozzi, il tipo di tubazioni per l'aria compressa, i compressori e l'attrezzatura per il controllo e il monitoraggio. In questo caso, le iniezioni sono state effettuate sia in verticale attraverso tubi piezometrici micro-fessurati distanziati l'un l'altro di $10 \mathrm{~m}$, sia in orizzontale (ad un metro di profondità) in corrispondenza della zona con maggiore concentrazione di inquinanti. In particolare, sono stati utilizzati $50 \mathrm{Kg}$ di bioattivatore con $130 \mathrm{Kg}$ di nutrienti nei piezometri verticali e $20 \mathrm{Kg}$ di bioattivatore con $60 \mathrm{Kg}$ di nutrienti nei tubi orizzontali. Negli stessi piezometri, è stata iniettata aria a bassa pressione (1 bar) tramite una soffiante.

Nella fase di start-up è fondamentale valutare la profondità della falda, la pressione e i livelli di ossigeno disciolti per accertarsi che le condizioni siamo ottimali. Successivamente, bisogna monitorare settimanalmente i livelli dei contaminanti e quelli dell'ossigeno per valutare l'efficienza del processo ed arrivare alle concentrazioni desiderate. Nel caso specifico, si sono eseguiti controlli dopo 30, 90 e 180 giorni dall'inoculo sia nella matrice suolo e sottosuolo che nella falda. Una volta rientrati nei valori delle Csc, si è proceduti al collaudo finale ed al monitoraggio semestrale (per una durata totale di 24 mesi) per valutare la conformità degli obiettivi di bonifica.

\section{Conclusioni}

In conclusione, il biosparging ha consentito il raggiungimento di ottimi risultati di abbattimento dei contaminanti sia per il terreno che per la falda. In particolare, la riduzione di idrocarburi leggeri, toluene e benzoatracene al di sotto dei limiti normativi, si è registrata dopo soli trenta giorni dall'inizio del trattamento; gli idrocarburi pesanti e gli idrocarburi totali, invece hanno necessitato rispettivamente di novanta e centottanta giorni. In definitiva, i valori delle concentrazioni al collaudo sono risultati ben al di sotto dei limiti normativi nonostante i tempi rapidi. Questi risultati confermano l'efficienza del processo di biosparging e rappresentano un'opportunità per le prossime applicazioni di bioremediation di siti contaminati. 


\section{References}

1.Carrè, F. et al.. Soil Contamination and Human Health: A Major Challenge for Global Soil Security. in Progress in Soil Science 275-295 (Springer International Publishing, 2017). doi:10.1007/978-3-319-43394-325.

2.Panagos, P., Liedekerke, M. V., Yigini, Y. \& Montanarella, L. Contaminated Sites in Europe: Review of the Current Situation Based on Data Collected through a European Network. Journal of Environmental and Public Health 2013, 1-11 (2013).

3.Brignoli, P., Martino, M. D. \& Gagliardi, G. Bioventing e Biosparging in area contaminata da TPH e PAHs. in SiCon 2014 Workshop su: Siti Contaminati. Esperienze negli interventi di risanamento (2014).

4.Chaineau, C. H., Yepremian, C., Vidalie, J. F., Ducreux, J. \& Ballerini, D. Bioremediation of a Crude Oil-Polluted Soil: Biodegradation, Leaching and Toxicity Assessments. Water Air, and Soil Pollution 144, 419-440 (2003).

5.Cappucci, S., Carloni, A. \& Maffucci, M. Tecnologie per la bonifica e riqualificazione. SOSTENIBILITÀ DEI SISTEMI PRODUTTIVI - Strumenti e tecnologie verso la green economy vol. 8 337-363 (Enea, 2012).

6.Azubuike, C. C., Chikere, C. B. \& Okpokwasili, G. C. Bioremediation techniques-classification based on site of application: principles advantages, limitations and prospects. World Journal of Microbiology and Biotechnology 32, (2016).

7.Kao, C. M., Chen, C. Y., Chen, S. C., Chien, H. Y. \& Chen, Y. L. Application of in situ biosparging to remediate a petroleum-hydrocarbon spill site: Field and microbial evaluation. Chemosphere 70, 1492-1499 (2008).

8.Machackova, J., Wittlingerova, Z., VIk, K. \& Zima, J. Major factors affecting in situ biodegradation rates of jet-fuel during large-scale biosparging project in sedimentary bedrock. Journal of Environmental Science and Health Part A 47, 1152-1165 (2012).

9.Khudur, L. S. et al.. Evaluating the efficacy of bioremediating a dieselcontaminated soil using ecotoxicological and bacterial community indices. Environmental Science and Pollution Research 22, 14809-14819 (2015).

10. Hatzinger, P. B. \& Lippincott, D. R. Field demonstration of NNitrosodimethylamine (NDMA) treatment in groundwater using propane biosparging. Water Research 164, 114923 (2019).

11.Lippincott, D. et al.. Bioaugmentation and Propane Biosparging for In Situ Biodegradation of 1,4-Dioxane. Groundwater Monitoring \& Remediation 35, 81-92 (2015). 
12.Sui, H. \& Li, X. Modeling for Volatilization and Bioremediation of Toluene-contaminated Soil by Bioventing. Chinese Journal of Chemical Engineering 19, 340-348 (2011).

13. Höhener, P. \& Ponsin, V. In situ vadose zone bioremediation. Current Opinion in Biotechnology 27, 1-7 (2014).

14.Thomè, A., Reginatto, C., I.Cecchin \& Colla, L. M. Bioventing in a Residual Clayey Soil Contaminated with a Blend of Biodiesel and Diesel Oil. Journal of Environmental Engineering 140, 06014005 (2014).

15.Verma, J. P. \& Jaiswal, D. K. Book Review: Advances in Biodegradation and Bioremediation of Industrial Waste. Frontiers in Microbiology 6 , (2016). 